\title{
Molecular Dynamics Simulation of the Spontaneous Formation of a Small DPPC Vesicle in Water in Atomistic Detail
}

\author{
Alex H. de Vries, ${ }^{*}$ Alan E. Mark, and Siewert J. Marrink \\ Department of Biophysical Chemistry, University of Groningen, Nijenborgh 4, \\ 9747 AG Groningen, The Netherlands
}

Received November 28, 2003; E-mail: a.h.de.vries@chem.rug.nl

Molecular dynamics (MD) simulations have been used to study the spontaneous aggregation of a concentrated solution of dipalmitoylphosphatidylcholine (DPPC) molecules in water in atomistic detail. Starting from a random solution of DPPC in water, an oblong-shaped vesicle with a long axis of $15 \mathrm{~nm}$ and short axes of $10 \mathrm{~nm}$ formed spontaneously.

Phospholipid vesicles (liposomes) are studied widely as model systems for biological membranes. ${ }^{1}$ Elucidating the mechanisms by which these bodies form, change shape, fuse, bud, and dissolve is central to understanding a wide range of biological phenomena and is hence the subject of intensive research both experimentally and theoretically. ${ }^{2}$

Previous simulation studies using simplified models have shown that, under appropriate conditions, small vesicles can form spontaneously from disordered solutions of amphiphiles. ${ }^{3}$ Here, we report the simulation of the spontaneous formation of a small vesicle from a disordered solution of DPPC in water in atomistic detail. Atomistic detail in these calculations allows one to (i) analyze the nature of intermediate structures in detail, (ii) assess the relevance of simulating small patches of a bilayer as a means of understanding the properties of liposomes, ${ }^{4}$ (iii) judge the reliability of earlier simulations performed using coarse-grained (CG) models, ${ }^{3}$ and (iv) analyze the effects of curvature which may be important in understanding processes such as vesicle fusion. ${ }^{5}$

The DPPC force field has been described previously. ${ }^{6}$ The DPPC molecule is represented by 50 interaction sites. Aliphatic $\mathrm{H}$-atoms are not treated explicitly, but are treated as one site with the $\mathrm{C}$-atom they are attached to. The three-site simple point charge (SPC) model was used to describe the water. The simulation conditions were the same as those used previously. ${ }^{6}$

A starting structure of a DPPC solution was prepared in two steps. First, 1017 DPPC molecules were distributed randomly in a cubic box (edge length $14.7 \mathrm{~nm}$ ). Next, the box was enlarged by $1.3 \mathrm{~nm}$ in each dimension and 106563 water molecules were added. Overall, the water:lipid ratio was 100:1. The water:lipid ratio in the DPPC-rich region was 32:1. The aim of creating a layer of water around the DPPC-rich region was to reduce the probability that any DPPC aggregate would fuse with its periodic image and thus form a lamellar phase. ${ }^{7}$ The formation of other types of aggregates, such as a vesicle, might thus be promoted. ${ }^{8}$

Figure 1 shows a series of snapshots illustrating the evolution of the DPPC:water mixture during a $90 \mathrm{~ns}$ MD simulation. The (random) starting solution is shown in Figure 1A. The region of high DPPC concentration is clearly separated from its periodic images by a layer of water. The initial stages of the aggregation process are very rapid. First, micellar-like structures form which, because of the high concentration of DPPC, are very tightly packed. The structure after $1 \mathrm{~ns}$, which is best described as a collection of associated micellar structures, is shown in Figure 1B. Lipids that bridge between these micelles lead to the creation of larger, lamellar aggregates as shown in Figure 1C (5 ns). The lamellar structures are in essence curved bilayers. Curvature develops spontaneously as a consequence of the minimization of edge energy. ${ }^{1}$ The increase in the curvature of the larger lamellar structures leads to the overall aggregate adopting a vesicle-like shape. This is already visible within $30 \mathrm{~ns}$ (Figure 1D). The gradual optimization of the vesicle is much slower than the initial aggregation. After $90 \mathrm{~ns}$ (Figure $1 \mathrm{E})$, the overall structure is clearly that of a vesicle. Nevertheless, there remain seven water pores, ranging in diameter between 1 and $4 \mathrm{~nm}$, two of which are visible in Figure 1E. These water pores are similar to those observed in simulations of the spontaneous aggregation of lipids into bilayers, ${ }^{7}$ and of induced pore-formation in bilayers through the application of a lateral tension or of an electric field. ${ }^{9}$

The collapse of such water pores is the rate-limiting step in the spontaneous formation of bilayers ${ }^{7}$ and has been found to take between 5 and $50 \mathrm{~ns}$. Sealing the vesicle may require much longer simulation time. Due to the high curvature, the vesicle wall is under stress. This may stabilize water pores as suggested by electroporation experiments. ${ }^{10}$ Also, the size of the vesicle is at the lower limit of DPPC vesicles observed experimentally. ${ }^{11}$

In the vesicle, the water pores facilitate the equilibration of lipids between the inner and outer leaflets as shown in Figure 2. This exchange appears to be a multistep process. Lipids are seen to diffuse into the pore. The headgroup of a lipid may reside in the pore for an extended period of several nanoseconds. During this time, the lipid tails rearrange. The lipid headgroup may then diffuse out of the pore region into a lamellar region.

Comparing the current work to our previous simulations of vesicle formation using a CG representation of DPPC (parametrized based on atomistic simulations), ${ }^{3 \mathrm{~d}}$ we find that the global features of the process of aggregation are similar. Due to a higher lipid density in the atomistic model, the intermediates are in general less well defined. Pores tend to be less stable in the CG model due to a higher line tension in that model. The bicelle-cup-vesicle transition as described for the CG model at lower concentration is not observed. ${ }^{12}$

The final structure of the vesicle (see Figure 3A) is similar to that found in the CG model. ${ }^{3 \mathrm{~d}}$ Approximately $28 \%$ of the lipids are in the inner leaflet. ${ }^{13}$ This is comparable to the $27-29 \%$ found using the CG model. As with the CG vesicles, the inner leaflet appears to be thinner than the outer leaflet. This is due to packing constraints which are a consequence of the high curvature. Order parameters of the lipid tail segments were calculated with respect to the local bilayer normal. These indicate that the tails of the lipids in the inner leaflet ( $S$ for methylene groups $2-6 \approx-0.17$ ) are significantly less ordered than those of the lipids in the outer leaflet $(S \approx-0.23)$. The order parameters of the lipid tails of the outer leaflet are similar to those of an equilibrated lamellar bilayer. Differences between lipids in the inner and outer leaflets are 


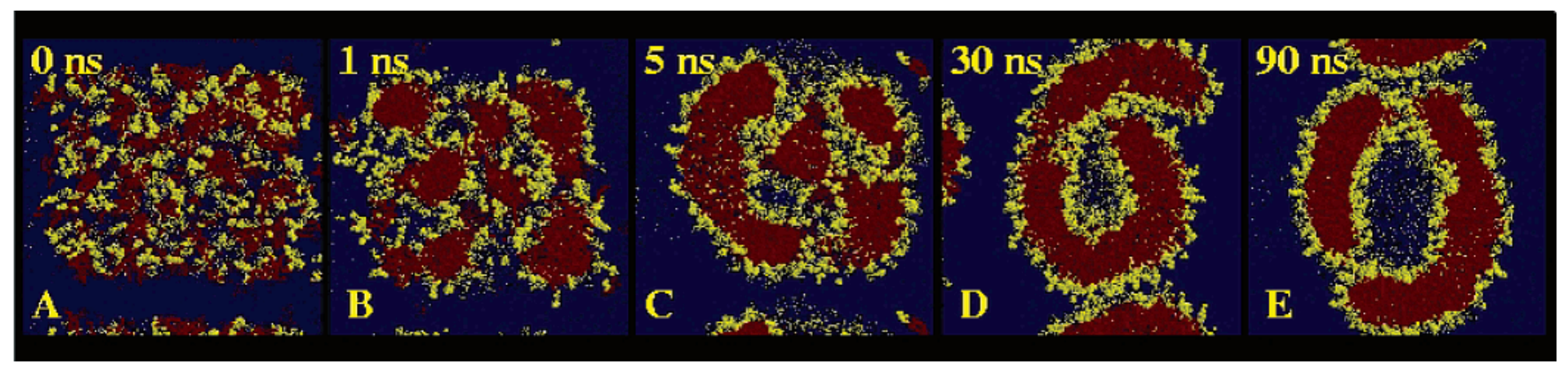

Figure 1. Snapshots of molecular dynamics simulation of lipid aggregation in water. Lipid headgroups (yellow); lipid tails (red); water (blue). Note that images correspond to transverse sections through the simulation box and that parts of the periodic images are also shown. Illustrated are (A) the starting structure, (B) a series of connected micelles, (C) curved bilayers, (D) vesicular aggregate, and (E) the final vesicle, showing two defects.

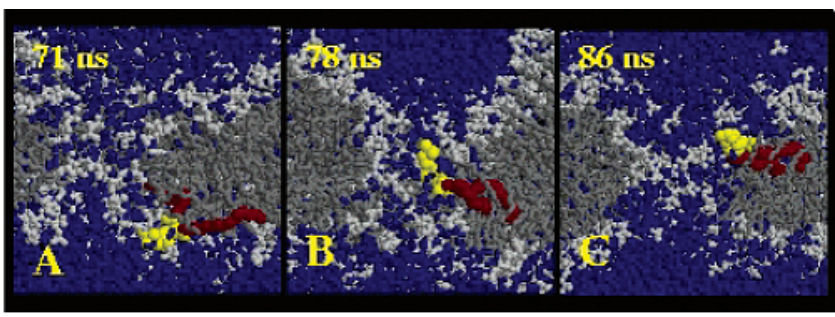

Figure 2. The movement of a lipid from the outer to the inner leaflet via a water pore. (A) The highlighted lipid first moves into the pore opening, (B) resides in the water pore for $15 \mathrm{~ns}$, before (C) finally moving to the inner leaflet.

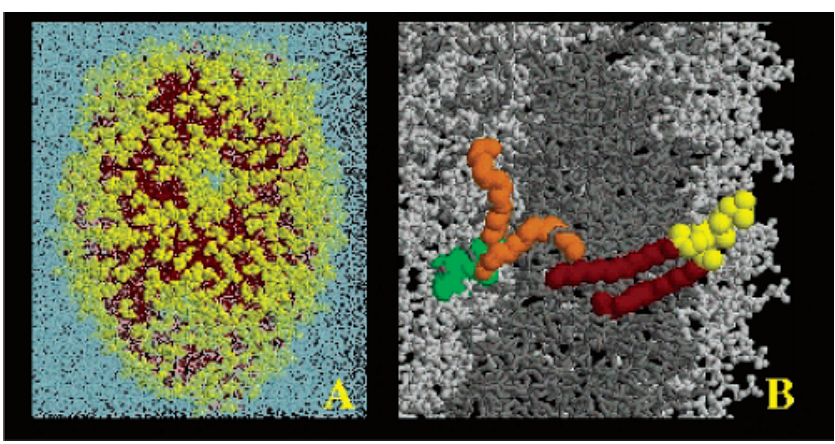

Figure 3. (A) The vesicle after $90 \mathrm{~ns}$ of simulation. Water is light blue. The lipids are colored as in Figure 1. The more intense blue near the center indicates a water pore. (B) Representative lipid conformations of lipids in the outer (yellow and red) and inner (green and orange) leaflets. Other lipids are shown in light gray (headgroups) and dark gray (tails), respectively. Water is not shown.

illustrated in Figure 3B. As can be seen, the tails of lipids in the outer leaflet (red) lie more along the local bilayer normal than the tails of lipids in the inner leaflet (orange). In contrast, the orientations of the headgroups with respect to each other are similar in the inner and outer leaflets. ${ }^{14}$ The density of the headgroups in the inner leaflet is, however, higher than that in the outer leaflet. Hence, the hydration of the outer leaflet headgroups is higher.

To summarize, we have demonstrated the possibility to simulate the spontaneous formation of a small lipid vesicle in atomistic detail using conventional MD simulation techniques. We find that in small vesicles with high curvature there are marked differences in the structural and dynamic properties between lipids in the inner and outer leaflets. Water pores facilitate transport of lipids between leaflets, which may hint at a more general pathway for transbilayer transport of amphiphiles. We also find that, while the general mechanism by which the vesicle forms is similar in simulations using CG and atomistic models, there are differences in the details of the process. Quantitative results concerning activated processes are expected to be different in the two models. As an example, we have observed that water pores appear to be more stable when the system is described in atomistic detail.

Acknowledgment. A.H.V. acknowledges MSC ${ }^{\text {lus }}$. S.J.M. is funded by the Royal Academy of Sciences (KNAW).

Supporting Information Available: Details of the force field and simulation setup. Illustrations of the structure of the vesicle. Local order parameter profile of lipid tails, radial distribution functions of selected atoms, and relative orientation of lipid headgroups (PDF). This material is available free of charge via the Internet at http://pubs.acs.org.

\section{References}

(1) Lasic, D. D. Liposomes: from physics to applications; Elsevier Science B.V.: Amsterdam, 1993

(2) Baumgart, T.; Hess, S. T.; Webb, W. W. Nature 2003, 425, 821-824

(3) (a) Drouffe, J. M.; Maggs, A. C.; Leibler, S. Science 1991, 254, 13531356. (b) Noguchi, H.; Takasu, M. Phys. Rev. E 2001, 64, 41913. (c) Yamamoto, S.; Maruyma, Y.; Hyodo, S. J. Chem. Phys. 2002, 116, 58425849. (d) Marrink, S. J.; Mark, A. E. J. Am. Chem. Soc. 2003, 125, $15233-$ 15242 .

(4) Scott, H. L. Curr. Opin. Struct. Biol. 2002, 12, 495-502.

(5) Kozlovsky, Y.; Kozlov, M. M. Biophys. J. 2002, 82, 882-895.

(6) Simulations were performed using isotropic pressure ( $P=1 \mathrm{bar})$ conditions at $T=323 \mathrm{~K}$ with the force field as described for system $\mathrm{F}$ in: Anézo, C.; de Vries, A. H.; Höltje, H.-D.; Tieleman, D. P.; Marrink, S. J. J. Phys. Chem. B 2003, 107, 9424-9433, with a long-range cutoff of $1.5 \mathrm{~nm}$, using a reaction-field correction and periodic boundary conditions. All simulations were performed with the GROMACS code (Lindahl, E.; Hess, B.; van der Spoel, D. J. Mol. Model. 2001, 7, 306-317) on a cluster of $1.7 \mathrm{GHz}$ Intel Pentium IV processors. The code was run in parallel on four or eight processors under MPI. The rate of simulation was 1 ps per processor CPU hour.

(7) Marrink, S. J.; Lindahl, E.; Edholm, O.; Mark, A. E. J. Am. Chem. Soc 2001, 123, 8638-8639.

(8) Note, the formation of a (multi)lamellar system is not prohibited

(9) (a) Tieleman, D. P.; Leontiadou, H.; Mark, A. E.; Marrink, S. J. J. Am. Chem. Soc. 2003, 125, 6382-6383. (b) Leontiadou, H.; Mark, A. E. Marrink, S. J. Biophys. J. 2004, in press.

(10) Kakorin, S.; Liese, T.; Neumann, E. J. Phys. Chem. B 2003, 107, $10243-$ 10251 .

(11) (a) Cornell, B. A.; Fletcher, G. C.; Middlehurst, J.; Separovic, F. Biochim Biophys. Acta 1982, 690, 15-19. (b) Vesicles with a diameter of $13 \mathrm{~nm}$ have been observed for other amphiphiles: Buwalda, R. T.; Stuart, M. C. A.; Engberts, J. B. F. N. Langmuir 2002, 18, 6507-6512.

(12) A simulation using the CG model of ref $3 \mathrm{~d}$ with the same setup as used for the atomistic model resulted in a sealed vesicle containing 1012 lipids after $30 \mathrm{~ns}$ with intermediates similar to those shown here for the atomistic model.

(13) The vesicular state at the end of the simulation is formed by 1002 DPPC lipids. The remaining 15 lipids formed a micelle during the simulation. The assignment of lipids to inner and outer leaflets is somewhat arbitrary because a number of headgroups are lining water pores and therefore do not clearly belong to either the inner or the outer leaflet.

(14) First neighbor headgroups strongly favor antiparallel orientations, minimizing dipole-dipole repulsion. Second neighbors show a weak preference for parallel orientation. After the second shell of neighbors, the correlation disappears quickly.

JA0398417 